\title{
A Verification Scenario of Nuclear Plus Interference Scattering Effects Using Neutron Incident Angle Distribution to the Wall in Beam-Injected Deuterium Plasmas*
}

\author{
Shota SUGIYAMA, Hideaki MATSUURA, Daisuke Uchiyama, Daisuke SAWADA, \\ Tsuguhiro WATANABE ${ }^{1)}$, Osamu MITARAI ${ }^{2)}$ and Takuya GOTO ${ }^{1)}$ \\ Department of Applied Quantum Physics and Nuclear Engineering, \\ Kyushu University, 744 Motooka, Fukuoka 819-0395, Japan \\ 1) National Institute for Fusion Science, 322-6 Oroshi-cho, Toki 509-5292, Japan \\ ${ }^{2)}$ Kumamoto Liberal Arts Education Center, Tokai University, 9-1-1 Toroku, \\ Higashiku, Kumamoto 862-8652, Japan
}

(Received 25 November 2014 / Accepted 2 February 2015)

\begin{abstract}
A verification scenario of knock-on tail formation in the deuteron distribution function due to nuclear plus interference scattering is presented by observing the incident angle distribution of neutrons in a vacuum vessel. Assuming a knock-on tail created in a ${ }^{3} \mathrm{He}$-beam-injected deuterium plasma, the incident angle distribution and energy spectra of the neutrons produced by fusion reactions between $1-\mathrm{MeV}$ and thermal deuterons are evaluated. The relation between the neutron incident angle to the vacuum vessel and neutron energy is examined in the case of anisotropic neutron emission due to knock-on tail formation in neutral-beam-injected plasmas.
\end{abstract}

(c) 2015 The Japan Society of Plasma Science and Nuclear Fusion Research

Keywords: nuclear plus interference scattering, fast-ion velocity distribution function, neutron emission spectrum, beam-injected deuterium plasma, neutron incident angle distribution, neutron incident energy spectrum, Large Helical Device

DOI: $10.1585 /$ pfr. 10.3403055

\section{Introduction}

Energetic ions contribute to form knock-on tails in ion velocity distribution functions [1-3] due to nuclear plus interference (NI) scattering ${ }^{1}[4,5]$. As a consequence of knock-on tail formation, the neutron emission spectrum is modified from a Gaussian distribution [1,6-8]. It is well known that the fraction of transferred power from the energetic to bulk ions is increased by NI scattering $[9,10]$. In such a case, the required power fraction to maintain the hot ion mode was obtained in a $\mathrm{D}-{ }^{3} \mathrm{He}$ spherical tokamak reactor [11]. By detecting the non-Gaussian component in the neutron emission spectrum, the knock-on tail and NI scattering effects can be diagnosed. Previously, possible verification scenarios for knock-on tail formation have been studied using the neutron emission spectrum [7,8]. However, the non-Gaussian component is several orders of magnitude less than the peak of the Gaussian distribution function; hence, it is susceptible to the effects of background noise.

In deuterium plasmas, energetic protons are produced by the $\mathrm{D}(\mathrm{d}, \mathrm{p}) \mathrm{T}$ and ${ }^{3} \mathrm{He}(\mathrm{d}, \mathrm{p})^{4} \mathrm{He}$ reactions. These protons, especially by the latter reaction, cause large NI scat-

\footnotetext{
author'se-mail: s-sugi@nucl.kyushu-u.ac.jp

*) This article is based on the presentation at the 24th International Toki Conference (ITC24).

${ }^{1}$ NI scattering is defined by subtracting Coulomb contributions from experimental data. It is also referred to as "nuclear elastic scattering (NES)".
}

tering and contribute to the knock-on tail formation in the deuteron velocity distribution function [9-12] (modification of the neutron emission spectrum [8]). If a small amount of ${ }^{3} \mathrm{He}$ is externally admixed, the knockon grows large because the proton production rate by the ${ }^{3} \mathrm{He}(\mathrm{d}, \mathrm{p})^{4} \mathrm{He}$ reaction is enhanced. The degree of enhancement of the $\mathrm{D}(\mathrm{d}, \mathrm{p}) \mathrm{T}, \mathrm{T}(\mathrm{d}, \mathrm{n}){ }^{4} \mathrm{He}$, and ${ }^{3} \mathrm{He}(\mathrm{d}, \mathrm{p})^{4} \mathrm{He}$ fusion reaction rate coefficient in a ${ }^{3} \mathrm{He}$-beam-injected deuterium plasma has been evaluated [13].

In neutral-beam-injected (NBI) plasmas, the knockon tail in the ion velocity distribution function and the non-Gaussian component in the neutron emission spectrum have anisotropic distributions because beam particles are injected in a particular direction. An effect of the anisotropy of emitted neutrons appears when they collide with the first wall. In toroidal devices, even for an isotropic emission of the neutrons, it is well known that the neutron flux and wall loading depend on wall positions $[14,15]$. Because incident neutrons produced by suprathermal reactions have an anisotropic spectrum, the incident directions of neutrons to the first wall are also anisotropic when the knock-on tail is formed. By grasping the combination of the incident angle and the wall position where the largest number of non-Gaussian neutrons is observed, we can determine the most suitable detector position and angle for the knock-on tail diagnosis. This trend could be seen in all 
toroidal devices. In addition to the previous scenario [8], we can obtain more detailed information to validate the knock-on tail formation due to NI scattering and its effects by looking at the neutron incident angle distribution to the first wall.

In this paper, we assumed a knock-on tail created in a ${ }^{3} \mathrm{He}$-beam-injected deuterium plasma confined in the Large Helical Device (LHD). The neutron incident angle distribution and energy spectrum produced by the reactions between $1-\mathrm{MeV}$ and thermal deuterons to the first wall are evaluated using a kinetic model combined with orbit calculations. A correlation between the neutron energy and incident angle, and a scenario to verify the NI scattering effects using the neutron incident angle distribution are presented.

\section{Analysis Model}

We assumed that the ${ }^{3} \mathrm{He}$ beam is tangentially injected at the magnetic axis $R_{\mathrm{ax}}=3.6 \mathrm{~m}$, and that the knock-on tail in the deuteron velocity distribution function is formed in the beam-injected direction. The deuteron velocity distribution is adopted from Ref. [8]. The density of $1-\mathrm{MeV}$ deuterons is about $2 \times 10^{11} \mathrm{~m}^{-3} \mathrm{keV}^{-1}$ when a $100-\mathrm{keV}$ ${ }^{3} \mathrm{He}$ beam is injected in the deuterium plasma. The bulkdeuteron density $n_{\mathrm{d}}=8 \times 10^{19} \mathrm{~m}^{-3}$, and the electron temperature $T_{\mathrm{e}}=20 \mathrm{keV}$ were assumed. The adopted deuteron distribution function is shown in Fig. 1.

The positions and directions of energetic deuteron at the instant when the $\mathrm{D}(\mathrm{d}, \mathrm{n})^{3} \mathrm{He}$ reaction occurs are calculated by the orbit calculation with the LHD field $B_{\text {ax }}$ $=2.74 \mathrm{~T}$. The cross section for the $\mathrm{D}(\mathrm{d}, \mathrm{n})^{3} \mathrm{He}$ reaction is taken from the work of Bosch et al. [16]. The orbit of a $1-\mathrm{MeV}$ deuteron for 200 toroidal turns after it is generated at the magnetic axis with pitch angle $0^{\circ}$ is used for this calculation as a test particle. The orbit for 10 toroidal turns in (a) the meridional plane and (b) the equatorial plane is shown in Fig. 2.

Neutron emission direction and energy are calculated using the positions, directions, and energies of the energetic deuterons. The neutron emission energy by the $\mathrm{D}(\mathrm{d}, \mathrm{n})^{3} \mathrm{He}$ reaction in the laboratory system is written as follows [17]:

$$
\begin{aligned}
E_{\mathrm{n}}= & \frac{1}{2} m_{\mathrm{n}} v_{0}^{2}+\frac{m^{3} \mathrm{He}}{m_{3^{3} \mathrm{He}}+m_{\mathrm{n}}}\left(Q+E_{\mathrm{r}}\right) \\
& +v_{0} \cos \zeta \sqrt{\frac{2 m_{3^{H e}} m_{\mathrm{n}}}{m_{3 \mathrm{He}}+m_{\mathrm{n}}}\left(Q+E_{\mathrm{r}}\right)},
\end{aligned}
$$

where $m_{\mathrm{n}\left({ }^{3} \mathrm{He}\right)}$ is the neutron $\left({ }^{3} \mathrm{He}\right)$ mass, $v_{0}$ is the centerof-mass velocity of the reacting deuterons, $\zeta$ is the angle between the center-of-mass velocity and neutron velocity vectors in the center-of-mass system, $Q$ is the reaction $Q$-value, and $E_{\mathrm{r}}$ is the relative energy of the reacting deuterons.

Neutron collision points with the first wall and neutron incident angles are calculated using the neutron emission direction and a mathematical expression of the shape

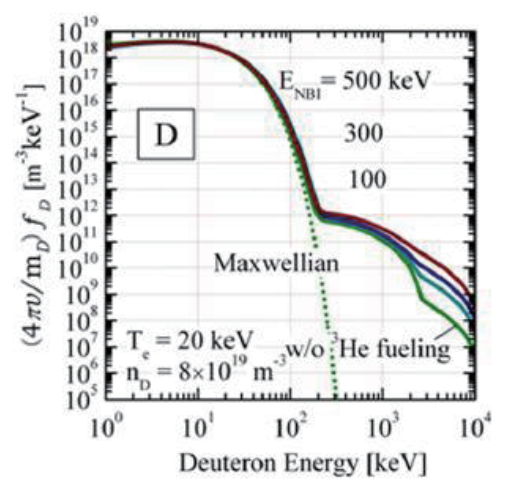

Fig. 1 Adopted deuteron distribution function [8]. The bulkdeuteron density $n_{\mathrm{d}}=8 \times 10^{19} \mathrm{~m}^{-3}$ and the electron temperature $T_{\mathrm{e}}=20 \mathrm{keV}$ are assumed.
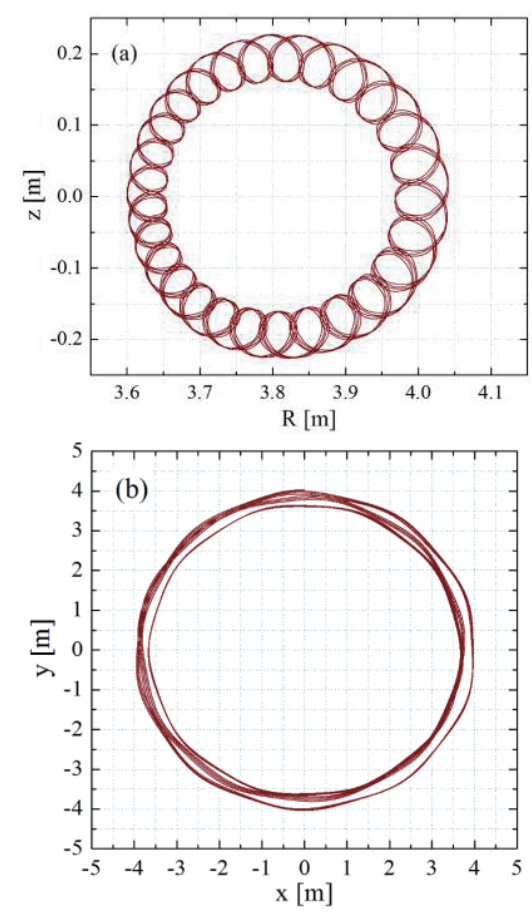

Fig. 2 Orbit of a 1-MeV deuteron for 10 toroidal turns in (a) the meridional plane, (b) the equatorial plane.

of the first wall. In this calculation, the shape of the vacuum vessel of the LHD as the first wall is assumed as a torus. The incident angle of the neutron to the first wall is calculated as the angle between the neutron emission direction and wall surface. We defined the incident angle in the meridional plane as the poloidal incident angle $\iota_{\mathrm{p}}$, and in the equatorial plane as the toroidal incident angle $\iota_{\mathrm{t}}$. The assumed first wall shape and defined incident angles in (a) the meridional plane and (b) the equatorial plane are shown in Fig. 3.

We assumed that neutrons by reactions of thermal deuterons are isotropically emitted from the inside of a torus with a major radius of $3.6 \mathrm{~m}$ and a minor radius of $0.3 \mathrm{~m}$. 


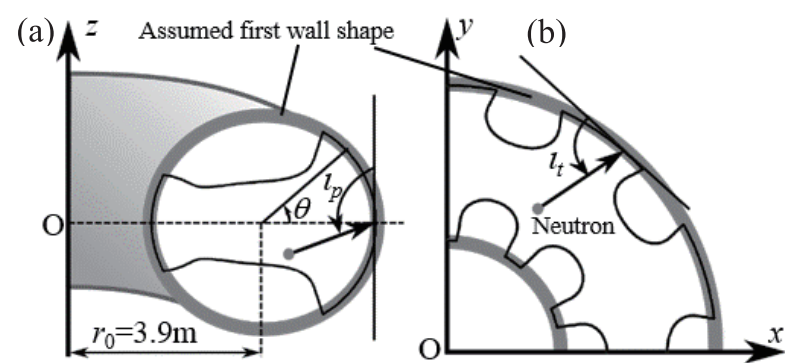

Fig. 3 Relations between the first wall shape and (a) the poloidal incident angle $\iota_{\mathrm{p}}$ in the meridional plane and (b) the toroidal incident angle $\iota_{\mathrm{t}}$ in the equatorial plane.

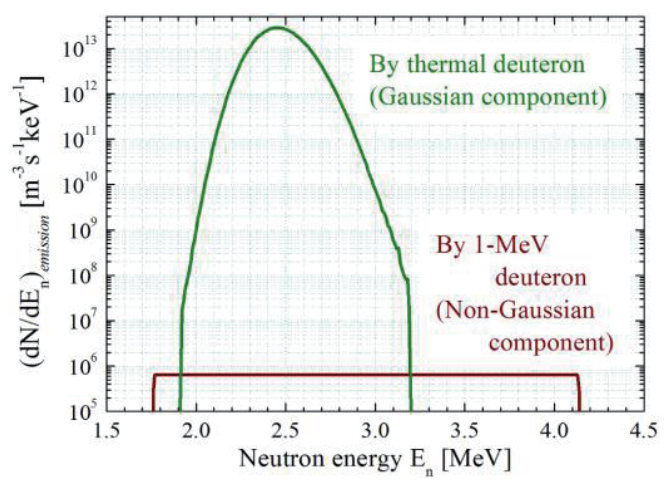

Fig. 4 Neutron emission spectrum by the $D(d, n)^{3} \mathrm{He}$ reaction when the knock-on tail is formed in the deuteron distribution function.

\section{Results and Discussion}

\subsection{Neutron emission spectrum}

The neutron emission spectrum by the $\mathrm{D}(\mathrm{d}, \mathrm{n})^{3} \mathrm{He}$ reaction is shown in Fig. 4. It is found that the non-Gaussian component is formed in the neutron emission spectrum with a range of energies from about 1.8 to $4.1 \mathrm{MeV}$, which is caused by the knock-on tail component in the deuteron velocity distribution function. The non-Gaussian component is about seven orders of magnitude smaller than the peak of the Gaussian component. However, because the non-Gaussian component has an anisotropic spectrum, the observed spectrum depends on the position and direction of the detector.

\subsection{Neutron incident energy spectrum to the wall}

The neutron incident energy spectra at (a) all wall positions and (b) $\theta=0^{\circ}$ are shown in Fig. 5. It is found that the neutron incident spectrum depends on the wall position. At wall position $\theta=0^{\circ}$, neutrons with a range of energies from about 1.8 to $4.1 \mathrm{MeV}$ are observed. As can be seen from Eq. (1), the neutron emitted in the same direction as the center-of-mass velocity of the reacting deuterons has the maximum energy, and the neutrons emitted in the opposite direction has the minimum energy. As the ${ }^{3} \mathrm{He}$ beam is injected tangentially in the equatorial plane and the
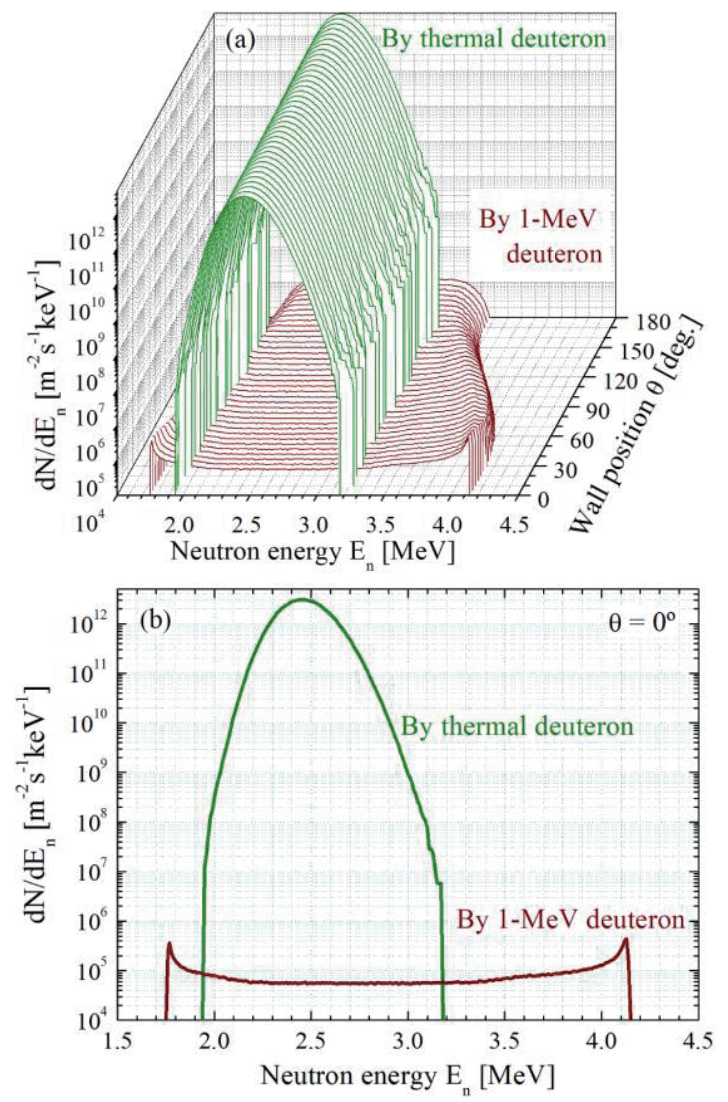

Fig. 5 Neutron incident energy spectra at (a) all wall positions, (b) $\theta=0^{\circ}$.

knock-on tail is formed in the beam-injected direction, the neutrons that have maximum and minimum energies can be observed only at $\theta=0^{\circ}$. In tangentially beam-injected plasmas, it can be said that the wall position $\theta=0^{\circ}$ is the most suitable for neutron measurements aimed at verifying knock-on tail formation, because this position observes the neutrons with a full range of energies in the non-Gaussian component.

\subsection{Neutron incident angle distribution to the wall}

The toroidal incident angles and energy distributions of neutrons at $\theta=0^{\circ}$ at (a) all toroidal incident angles and (b) $\iota_{\mathrm{t}}=45^{\circ}$ point are shown in Fig. 6 . The neutron incident angle correlates closely with the neutron emission energy and direction. It is recognized that two peaks exist at about 1.8 and $4.1 \mathrm{MeV}$. The neutron incident angle is determined by the geometric relationship between the shape of the first wall and the neutron emission direction. Neutrons emitted in the same direction as the center-of-mass velocity of the reacting deuterons, collide with the first wall at an incident angle $\iota_{\mathrm{t}}=45^{\circ}$ and with maximum energy. In the same manner, neutrons with the minimum energy collide with the first wall at an incident angle $\iota_{\mathrm{t}}=135^{\circ}$.

At $\iota_{\mathrm{t}}=45^{\circ}$, the largest number of neutrons in the nonGaussian component is observed and the non-Gaussian component is about five orders of magnitude smaller than 

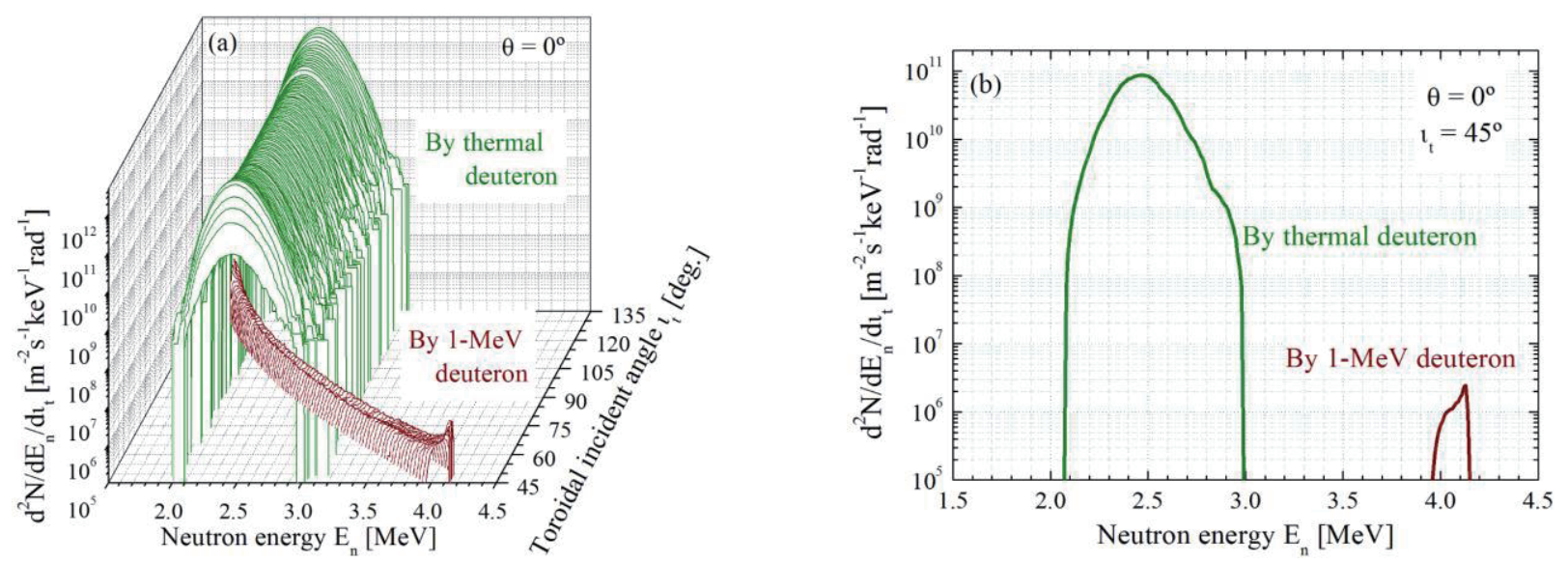

Fig. 6 Neutron incident energy spectra at the wall position $\theta=0^{\circ}$ for (a) all toroidal incident angles, (b) $\iota_{\mathrm{t}}=45^{\circ}$.

2.5-MeV peak. The largest number of neutrons in the Gaussian component in the neutron emission spectrum is observed at the incident angles of $50^{\circ}$ and $130^{\circ}$. When neutrons are isotropically emitted from a circumference with a radius of $3.6 \mathrm{~m}$, they can collide with the first wall with incident angles within the interval of about $49^{\circ} \leq \iota_{\mathrm{t}} \leq$ $131^{\circ}$. Therefore, it is difficult to observe neutrons by reactions of thermal deuterons at $\iota_{\mathrm{t}}=45^{\circ}$. If we observe the neutron spectrum at $\iota_{\mathrm{t}}=45^{\circ}$ point, the difference between the non-Gaussian and $2.5-\mathrm{MeV}$ Gaussian peak is reduced by two orders of magnitude compared with the space-averaged neutron emission spectrum. This is advantageous for improving the precision of fast-ion diagnostics.

\section{Conclusions}

By assuming a knock-on tail is formed in a ${ }^{3} \mathrm{He}$-beaminjected deuterium plasma confined in the LHD, a scenario to validate the NI scattering effects using the incident angle distribution and energy spectrum of neutrons is presented. By examining the dependence of the neutron energy on the incident angle to the first wall for fusion reactions between $1-\mathrm{MeV}$ and thermal deuterons, we found the most suitable position and angle for the neutron detector for diagnostics of knock-on tail formation. Furthermore, the difference between the non-Gaussian component and 2.5-MeV Gaussian peak in the neutron spectrum can be reduced by two orders of magnitude compared with the neutron emission spectrum.

In this paper, as a first step, it is shown that the differentiation of the neutron emission spectrum with respect to the incident angle is effective for knock-on tail diagnostics. The density, temperature, and spatial distribution of thermal deuteron are somewhat different from the LHD plasmas, because the calculation was made with reference to previous results [8]. To verify the NI scattering effects experimentally, the neutron incident angle and energy distribution should be evaluated considering the deuteron velocity distribution function with a full range of energies in the actual conditions of the LHD plasma. As the difference between the non-Gaussian component and 2.5-MeV Gaussian peak in the neutron emission spectrum is about four orders of magnitude [8], it is expected that the difference could be reduced to two orders of magnitude. A more detailed calculation with modifications to the analysis model (to consider the spatial and pitch-angle distributions of energetic deuteron and the actual shape of vacuum vessel of the LHD, etc.) is the subject of a future study.

[1] L. Ballabio et al., Phys. Rev. E 55, 3358 (1997).

[2] H. Matsuura et al., Phys. Plasmas 13, 062507 (2006).

[3] H. Matsuura et al., Plasma Phys. Control. Fusion 53, 035023 (2011).

[4] J.J. Devaney et al., Nucl. Sci. Eng. 46, 323 (1971).

[5] S.T. Perkins et al., Nucl. Sci. Eng. 77, 20 (1981).

[6] J. Källne et al., Phys. Rev. Let. 85, 1246 (2000).

[7] H. Matsuura et al., Fusion Sci. Technol. 60, 630 (2011).

[8] H. Matsuura et al., Plasma Fusion Res. 8, 2403064 (2013).

[9] J. Galambos et al., Nucl. Fusion 24, 739 (1984).

[10] Y. Nakao et al., Nucl. Fusion 28, 1029 (1988).

[11] O. Mitarai et al., Fusion Eng. Des. 81, 2719 (2006).

[12] H. Matsuura et al., Plasma Fusion Res. 7, 2403076 (2012).

[13] H. Matsuura et al., Plasma Fusion Res. 9, 3402062 (2014).

[14] P.P.H. Wilson et al., Fusion Eng. Des. 83, 824 (2008).

[15] J.C. Rivas et al., Fusion Sci. Technol. 64, 687 (2013).

[16] H.-S. Bosch et al., Nucl. Fusion 32, 611 (1992).

[17] H. Brysk, Plasma Phys. 15, 611 (1973). 